STUDI

FRANCESI

\section{Studi Francesi}

Rivista quadrimestrale fondata da Franco Simone

165 (LV | III) | 2011

LA RAPPRESENTAZIONE DELLA MADRE NELLA

LETTERATURA FRANCESE DEL NOVECENTO a cura di Dario Cecchetti e Michele Mastroianni

\title{
Ann Thomson, Simon Burrows, Edmond Dziembowski (a cura di), Cultural transfers: France and Britain in the long eighteenth century
}

\section{Marisa Ferrarini}

\section{(2) OpenEdition}

\section{Journals}

\section{Edizione digitale}

URL: https://journals.openedition.org/studifrancesi/5005

DOI: 10.4000/studifrancesi.5005

ISSN: 2421-5856

\section{Editore}

Rosenberg \& Sellier

\section{Edizione cartacea}

Data di pubblicazione: 1 décembre 2011

Paginazione: 638-639

ISSN: 0039-2944

\section{Notizia bibliografica digitale}

Marisa Ferrarini, «Ann Thomson, Simon Burrows, Edmond Dziembowski (a cura di), Cultural transfers: France and Britain in the long eighteenth century», Studi Francesi [Online], 165 (LV | III) | 2011, online dal 30 novembre 2015, consultato il 21 septembre 2021. URL: http://journals.openedition.org/ studifrancesi/5005; DOI: https://doi.org/10.4000/studifrancesi.5005

Questo documento è stato generato automaticamente il 21 septembre 2021.

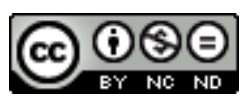

Studi Francesi è distribuita con Licenza Creative Commons Attribuzione - Non commerciale - Non opere derivate 4.0 Internazionale. 


\title{
Ann Thomson, Simon Burrows, Edmond Dziembowski (a cura di), Cultural transfers: France and Britain in the long eighteenth century
}

\author{
Marisa Ferrarini
}

\section{NOTIZIA}

ANN THOMSON, SIMON BURROWS, EDMOND DZIEMBOWSKI (a cura di), Cultural transfers: France and Britain in the long eighteenth century, Oxford, Voltaire Foundation, 2010, pp. 328.

Questo libro è il risultato del progetto triennale intitolato «Transferts culturels francobritanniques, fin $\mathrm{XVII}^{\mathrm{e}}$ au début $\mathrm{XIX}^{\mathrm{e}}$ siècle: agents, vecteurs, réseaux/ Franco-British cultural transfers in the long eighteenth century: agents, vectors, networks», diretto dal gruppo di ricerca «en histoire intellectuelle» dell'Università di Paris VIII, finanziato dall'Ambasciata di Francia in Gran Bretagna, dall'Agenzia Nazionale della Ricerca Francese e dal British Arts and Humanities Research Council.

2 Come appare chiaro sin dal titolo, si tratta di stabilire se gli scambi culturali tra Francia e Gran Bretagna abbiano contribuito a formare l'identità specifica della singola nazione o a fondare una «République des lettres», termine oggi molto alla moda grazie al saggio di Pascale Casanova (La République mondiale des lettres, Paris, Seuil, 1999, ripubblicato nel 2008), di cui nel testo non c'è tuttavia menzione. Ovviamente, i curatori optano per la seconda possibilità e giustamente s'interrogano sulle modalità della comunicazione. Quali canali? Come funzionavano? Che tipo d'impatto avevano?

Da qui la suddivisione tripartita e piuttosto aleatoria (visto che vari personaggi transitano da una sezione all'altra) in «Correspondence and networks», «Journalism» e «Translation». 
4 Nella prima parte, Rachel HAMMERSLEY (The "Real Whig"-Huguenot network and the English Republican tradition, pp. 19-32) mostra come gli esuli ugonotti dopo la revoca dell'Editto di Nantes, abbiano diffuso sul continente, attraverso pubblicazioni, traduzioni e riviste, le idee di una tradizione democratica. Elizabeth GRIST (Pierre Des Maizeaux and the Royal Society, pp. 33-42) focalizza l'attenzione sul legame che si stabilì tra il giornalista Pierre Des Maizeaux e la Royal Society per esportare in Olanda le teorie newtoniane attraverso la corrispondenza. Seguono interessanti articoli riguardanti la natura e la definizione di corrispondenza diplomatica (Charles-Édouard LEVILLAIN, La correspondance diplomatique dans l'Europe moderne: problèmes de méthode et tentative de définition (pp. 43-55), lo scambio internazionale di libri tra diplomatici (Joanna CRAIGWOOD, Diplomats and international book exchange, pp. 57-69), i rapporti massonici (Pierre-Yves BEAUREPAIRE, Quand les francsmaçons signent des traités diplomatiques: circulations et échanges maçonniques entre France et Angleterre (1765-1775), pp.71-84), il ruolo della comunità scientifica di Mont-pellier (James LIVESEY, London by the light of Mont-pellier: scientific networks between Britain, northern Europe and the Languedoc (1706-1789), pp. 85-102), le connessioni filantropiche (Mariana SAAD, Le réseau franco-britannique du «Recueil» Duquesnoy, pp. 103-114; JeanFrançois DUNYACH, Les réseaux d'un excentrique: vies et parcours de William Playfair (1759-1823), pp. 115-127).

5 La seconda parte verte sul ruolo svolto dalle gazettes, dai periodici specialistici e dai giornali sponsorizzati dal governo nella diffusione in Francia dei dibattiti politici e delle istituzioni inglesi. L'elenco degli articoli proposti è di per sé eloquente: Claire CRIGNONDE OLIVEIRA, Le rôle des périodiques dans la diffusion du savoir médical en France et en GrandeBretagne (fin dix-septième-fin dix-huitième siècle), pp.131-145; Delphine SoULARD, Les journalistes du Refuge et la diffusion de la pensée politique de John Locke auprès du public francophone dès la fin du dix-septième siècle, pp.147-159; Ann THomson, In defence of toleration: La Roche's "Bibliothèque angloise" and "Mémoires littéraires de la Grande-Bretagne", pp.161-174; Edmond DZIEMBOwSKI, Le peuple français instruit: Edme-Jacques Genet et la traduction des écrits politiques britanniques pendant la guerre de Sept ans, pp. 175-188; Simon BURROws, The "Courier de l'Europe» as an agent of cultural trans-fer (1776-1791), pp. 189-201.

6 La terza parte, forse per mia deformazione professionale, è senz'altro la più interessante. Stéphane ЈЕттот (La compréhension et la traduction des débats parlementaires à Londres par les diplomates de Louis XIV, pp. 205-218) delinea il ruolo del traduttore e dell'interprete negli incontri internazionali. Non avendo una padronanza della lingua inglese, poiché l'Inghilterra era considerata area culturale marginale, i diplomatici di Luigi XIV si affidarono, sulle prime, a esponenti delle élites inglesi che conoscevano, invece, perfettamente il francese. L'ingerenza crescente del Parlamento di Londra in politica estera dopo il 1660 li costrinse, però, a ricorrere a nuovi intermediari per capire i dibattiti che avevano luogo nell'assemblea. Nelle ambasciate comparvero così traduttori permanenti e si strinsero contatti con i rifugiati ugonotti e i librai londinesi. Ann Thомson (Des Maizeaux, Collins and translators: the case of Collins' "Philosophical inquiry concerning human liberty", pp. 219-231) mostra come una prima traduzione francese del saggio di Collins, pubblicata da Des Maizeaux nella sua collana nel 1720, non ebbe grande impatto in Francia e una seconda traduzione dal titolo diverso, nel 1754, ricevette uguale trattamento, per una serie di fraintendimenti, errori distributivi e mancanza di pubblicità. In Traduire, trahir et se trahir: le cas du "Pantheisticon" de John Toland (pp. 233-242), Pierre L URBE punta il dito sul problema dell'adattamento. La versione inglese e la versione francese dello stesso testo latino portano i segni 
dell'ambiente culturale al quale è destinato. Peraltro, le due traduzioni appaiono disomogenee: la semplice rappresentazione di fenomeni è un ostacolo epistemologico evidente nella traduzione francese. Chiudono la sezione tre articoli riguardanti la traduzione in Francia dei Political discourses di Hume (1752) e delle relative traduzioni nel 1754 (Michel MALHERBE, Hume en France: la traduction des "Political discourses", pp. 243-257); il ruolo svolto dalla Société typographique de Neuchâtel nel commercio traduttivo (Mark CURRAN, The Société typographique de Neuchâtel and networks of trade and translation in eighteenth-century francophone Europe, pp. 257-267); l'utilitarismo di Bentham (Emmanuelle DE CHAMPS, An introduction to utilitarianism: early French translations of Bentham's “Introduction to the principles of morals and legislation", pp. 269-283).

7 Anche se i diversi contributi affrontano solo una parte dei complessi rapporti che nel periodo preso in considerazione, e nella sua prima parte in modo del tutto particolare, si instaurarono tra Francia ed Inghilterra, il volume è tuttavia di grande interesse e contribuirà certamente a definire meglio l'interscambio culturale stabilitosi tra i due paesi. 